\title{
Algorithms for Routing and Wavelength Assignment Based on Solutions of LP-Relaxations
}

\author{
Rajesh M. Krishnaswamy and Kumar N. Sivarajan
}

\begin{abstract}
In this letter, we consider the problem of maximizing the number of lightpaths that may be established in a wavelength routed optical network (WRON), given a connection matrix, i.e., a static set of demands, and the number of wavelengths the fiber supports. The problem of establishing all the connections of the connection matrix using the fewest number of wavelengths has been investigated in [3], [6]. We call the former problem Max-RWA (problem of maximizing the number of lightpaths) and the latter problem Min-RWA (minimizing the number of wavelengths). In this letter, we only consider WRON's with no wavelength conversion capabilities. We formulate the Max-RWA problem when no wavelength conversion is allowed as an integer linear programme (ILP) which may be solved to obtain an optimum solution. We hope to solve the ILP exactly for small size networks (few nodes). For moderately large networks (tens of nodes) we develop algorithms based on solutions obtained by solving the LP-relaxation of the ILP formulation. Results obtained for networks such as NSFNET and EONNET are presented.
\end{abstract}

Index Terms-Linear programming, network, wavelength division multiplexing.

\section{INTRODUCTION}

W AVELENGTH routed optical networks (WRON's) are promising candidates for providing a flexible transport backbone network. The basic service which a WRON will offer is a lightpath service. A lightpath service between any two nodes of a WRON is the ability to provide a dedicated wavelength between them for carrying bits.

We consider the problem of maximizing the number of connections that can be established in a WRON (no wavelength changing) given a connection matrix and the number of wavelengths the fiber supports. This will be termed as the Max-RWA problem. The problem of realizing all the connections of a given connection matrix using the least number of wavelengths will be termed as the Min-RWA problem. The Min-RWA problem has been studied in detail in [3], [6]. Our methodology is to formulate the Max-RWA problem as an integer linear programme which when solved provides an optimal solution. We note that in [2], for the virtual topology design problem, we obtained solutions which were close to optimal when we rounded the solution

Manuscript received May 3, 2001. The associate editor coordinating the review of this letter and approving it for publication was Dr. J. Choe. This work was supported by a grant from the Department of Science and Technology, Government of India.

R. M. Krishnaswamy was with the Electrical and Commmunications Engineering Department, Indian Institute of Science Bangalore, India. He is now with Bell Labs, Lucent Technologies, Holmdel, NJ 07733 USA (e-mail: mkraj@home.com).

K. N. Sivarajan was with the Electrical and Communications Engineering Department, Indian Institute of Science Bangalore, India. He is now with Tejas Networks, Bangalore, India (e-mail: kumar@ tejasnetworks.com).

Publisher Item Identifier S 1089-7798(01)09861-1. obtained by solving the relaxed problem. This motivates us to use similar methods here too.

\section{A. Contribution of This Work}

In all the previous linear formulations for the Max-RWA problem (refer [1]) or the Min-RWA problem (refer [6]) the paths that the source-destination pair are allowed to take had to be specified beforehand. This is called as the path formulation ILP. As the number of paths in a graph between a node pair is exponential in the number of nodes of the graph so the path formulation will have to restrict itself to a few paths per node pair. When only a limited number of paths are considered the path formulation ILP approach may yield a sub-optimal solution.

Our linear formulation is a link based ILP formulation, i.e., the constraints are over the links (edges or arcs) of the network. The advantage of the above formulation is that we do not specify the paths before hand, but allow the integer linear program solver to choose any possible path and any possible wavelength for a source-destination pair and also the number of constraints in this formulation grow polynomially in the number of nodes. The link ILP formulation uses a set of constraints termed as wavelength continuity constraints. These constraints were first introduced in [2].

\section{PROBLEM Formulation: MAX-RWA}

In this section we present two 0-1 ILP formulations for the Max-RWA problem when no wavelength conversion is allowed. The first ILP formulation uses the wavelength continuity constraints of [2] and the second ILP formulation uses a new set of wavelength continuity constraints. The motivation for presenting the two formulations for the same problem stems from the fact that the two ILP's have different constraints sets and hence the heuristic algorithms, which takes as inputs the solution of LP-relaxations (illustrated in Section IV), may choose routes and wavelengths for source-destination pairs differently. We would like to compare the results obtained by rounding the solutions of the first ILP with that of the second ILP and come up with some criteria for choosing one heuristic over the other.

\section{A. First ILP Formulation}

Let $\rho_{(i, j)}$ denote the number of connections that are to be routed between node $i$ and node $j$. For each connection a route and a wavelength have to be assigned. We note that the wavelength and route assignment should be clash free, i.e., two lightpaths established using the same wavelength should not have 
any common physical link. The objective here is to maximize the number of carried connections.

We use the following notation. $i$ and $j$ denote originating and terminating node of a lightpath. $l$ and $m$ denote the endpoints of a physical link. $k$ when used as a superscript denotes the wavelength number. $q$ used as superscript or subscript denotes the $q$ th lightpath between a source-destination pair.

1) Parameters: $N=$ Number of nodes in the network. $\rho=$ the traffic matrix, i.e., $\rho_{i, j}$ is the number of connections that are to be established between node $i$ and node $j . P_{l m}$ denotes the existence of a link in the physical topology. If $P_{l m}=1$ then there is a fiber link between node $l$ and $m$, otherwise $P_{l m}$ is 0 . $F=$ the number of wavelengths the fiber can support.

2) Variables:

- $b_{q}(i, j)=1$, if there exists a $q$ th lightpath between $(i, j)$, else $b_{q}(i, j)=0$

- $C^{(k, q)}(i, j)=1$, if the $q$ th lightpath between node $i$ and node $j$ uses wavelength $k$; else $C^{(k, q)}(i, j)=0$.

- $C_{l, m}^{(k, q)}(i, j)=1$, if the $q$ th lightpath between node $i$ and node $j$ uses wavelength $k$ and is routed through physical link $(l, m)$; else $C_{l, m}^{(k, q)}(i, j)=0$.

Note that $k \in\{0,1,2,3, \ldots, F-1\}$, where $F$ is the number of wavelengths the fiber can support and for a given $(i, j), q \in$ $\left\{1,2,3 \ldots, \rho_{(i, j)}\right\}$.

3) Objective:

$$
\max \sum_{i, j} \sum_{q} b_{q}(i, j)
$$

Remark: Maximizes the number of connections.

4) Constraints:

$$
\sum_{q} b_{q}(i, j) \leq \rho_{i, j}, \quad \text { for all }(i, j) ; b_{q}(i, j) \in\{0,1\} .
$$

Remark: Ensures that number of connections established is atmost $\rho_{i, j}$.

- Wavelength continuity constraints

$$
\sum_{k=0}^{F-1} C^{(k, q)}(i, j)=b_{q}(i, j), \quad \text { for all } q \text { and }(i, j) \text {. }
$$

Remark: This ensures that if lightpath $b_{q}(i, j)$ exists then only one wavelength is assigned to it, among the $F$ possible choices

$$
C_{l, m}^{(k, q)}(i, j) \leq C^{(k, q)}(i, j) \text { for all } q,(i, j),(l, m) \text { and } k \text {. }
$$

Remark: The above equation ensures that only those $C_{l, m}^{(k, q)}(i, j)$ could be nonzero for which the corresponding $C^{(k, q)}(i, j)$ variables are nonzero

$$
\sum_{q} \sum_{(i, j)} C_{l, m}^{(k, q)}(i, j) \leq 1, \quad \text { for all } k \text { and }(l, m) .
$$

Remark: By the above constraint we ensure that no two lightpaths traversing through the physical link $(l, m)$ will have the same wavelength assigned to them

$$
\begin{aligned}
& \sum_{l} C_{l, m}^{(k, q)}(i, j) P_{l, m}-\sum_{l} C_{m, l}^{(k, q)}(i, j) P_{m, l} \\
& \quad= \begin{cases}C^{(k, q)}(i, j), & \text { if } m=i \\
-C^{(k, q)}(i, j), & \text { if } m=j ; \text { for all }(i, j), k, q \text { and } m, \\
0, & \text { if, } m \neq i \text { and } m \neq j .\end{cases}
\end{aligned}
$$

Remark: The above constraints ensure that the same wavelength is reserved at every node for a lightpath $b_{q}(i, j)$. Note that they analogous to the flow conservation equations in multicommodity flow problems.

Observation: In the first ILP, variables grow as $O\left(\sum \rho_{i, j} \times\right.$ Number of edges $\times F)$ and the number of constraints grows as $O\left(\sum \rho_{i, j} \times N \times F\right)$.

\section{B. Second ILP Formulation}

In this ILP formulation the constraints does not grow with the number of connections. Hence we may use this to solve the Max-RWA problem for larger networks.

We may think of a WRON with $F$ wavelengths being supported on a fiber as a network with $F$ multiple fibers links between the nodes with each fiber link supporting just one wavelength. Each of the links labeled $q \in\{0,1, \ldots, F-1\}$. We may now write the flow balance equations such that at each node the sum of the flows coming on links labeled $q$ is equal to the sum of the flows going out on the links labeled $q$. We observe that writing the flow balance equations for every label, $q$, captures wavelength continuity. The above observation motivates the subsequent ILP formulation.

1) Parameters: For sake of brevity we shall only enumerate any new terms used in this formulation.

- $P_{(l, m), q}=1$ denotes the existence of the $q$ th multiple link of the physical link $(l, m)$, otherwise $P_{(l, m), q}=0$. $q \in\{0,1,2,3 \ldots, F-1\}$.

2) Variables:

- $\lambda_{(l, m), q}^{(i, j)}=1$, if a lightpath setup between node $i$ and node $j$ uses the $q$ th multiple link of edge $(l, m)$ else $\lambda_{(l, m), q}^{(i, j)}=$ 0 .

- $b(i, j)$ denotes the number of lightpaths established between node $i$ and node $j$. It takes positive integral values.

3) Objective:

$$
\max \sum_{i} \sum_{j} b(i, j) .
$$

Remark: This maximizes the number of connections to be established.

4) Constraints:

$$
\begin{aligned}
\sum_{q} \sum_{m} \lambda_{(i, m), q}^{(i, j)} & =\sum_{q} \sum_{l} \lambda_{(l, j), q}^{(i, j)} \\
& =b(i, j) \leq \rho^{(i, j)} \quad \text { for all }(i, j) .
\end{aligned}
$$

Remark: The above set of equations ensures that the number of lightpaths from origin node $i$ and destination node $j$ are equal and is at most the requirement, $\rho^{(i, j)}$, for the source-destination pair $(i, j)$

$$
\begin{aligned}
& \sum_{l} \lambda_{(l, m), q}^{(i, j)} P_{(l, m), q}-\sum_{l} \lambda_{(m, l), q}^{(i, j)} P_{(m, l), q}=0 \\
& \quad \text { for all } q,(i, j), \text { and } m \neq i, m \neq j .
\end{aligned}
$$

Remark: This equation is a flow balance equation and ensures that at a node "wavelength is conserved," i.e., the same wavelength is used on the incoming and outgoing links of a lightpath

$$
\sum_{i} \sum_{j} \lambda_{(l, m), q}^{(i, j)} \leq 1 \quad \text { for all }(l, m), q .
$$


Remark: This ensures that there is at most one lightpath traversing link $\left(l, m_{)}, q\right.$.

Observation: In the second ILP, variables grow as $O(N(N-1) \times$ Number of edges $\times F)$ and the constraints grow as $O(N(N-1) \times N \times F)$. The second ILP has fewer constraints and variables than the first ILP.

\section{ALGORITHMS FOR MAX-RWA}

\section{A. First ILP and Second ILP}

For sake of brevity the we will only describe the algorithms here. A more detailed enumeration is in [4, Ch. 3]. Solution of the LP-relaxation yields fractional flows which when appropriately rounded up to a 0 or 1 yields an integer solution. For example when we solve the LP-relaxation of the first ILP we obtain variables $b_{q}(i, j) C^{k, q}(i, j)$ and $C_{l, m}^{k, q}(i, j)$ which contain the fractional flow information, where $C^{k, q}(i, j)=0.5$ would imply that 0.5 units of flow of the $q$ th connection of source-destination pair $(i, j)$ using color $k$ flows on physical link $(l, m)$.

We use two different approaches to obtain an integer solution. Algorithm-A rounds variables obtained by solving the first ILP. Note that by rounding variable of type $b_{q}(i, j), C^{k, q}(i, j)$, $C_{l, m}^{k, q}(i, j)$ we obtain a wavelength and a path to a connection of a source-destination pair $(i, j)$. This algorithm terminates when it can no longer round variables without violating wavelength continuity constraints.

Algorithm-B has two steps. In the first step the algorithm rounds the variables obtained by solving the relaxed problem, first ILP or second ILP, to get routes for all connections listed in the connection matrix. From the routes we derive a path-graph [1]. In the next step of the algorithm, we use heuristics to find a maximal set of nodes that can be vertex colored using $F$ colors. Note that this step is equivalent to finding the maximum number of connections that supported using $F$ wavelength [1].

\section{NUMERICAL RESULTS AND DISCUSSIONS}

\section{A. NSFNET and EONNET}

The skeleton of the NSFNET shown in [2] is a 14-node network with 21 edges. The European optical network (EONNET) shown in [5] is a 20-node network with 39 edges. In both these networks, each edge represents a pair of directed fibers, one in each direction.

The generation of connection matrix and the actual connection matrix used for NSFNET (total of 268 connections) and EONNET (total of 374 connections) is detailed in [4, Table 3.7 and Table 3.6] and [4, Table 3.6].

\section{B. Discussion of Results Obtained}

The results for NSFNET and EONNET are tabulated in Table I. We note that as we increase the number of available wavelengths beyond the LP lower bound (19 and 22) for NSFNET and EONNET, there is no marked improvement in the number of connections that are established. This may be explained as follows. If we solved the relaxed problem with a large number of wavelengths available then we observe that the fractional solutions obtained distributes over all possible routes and wavelengths available for a source-destination pair. This being the case the rounding heuristic which takes as inputs the
TABLE I

RESULTS OBTAINED FOR NSFNET AND EONNET

\begin{tabular}{|c|c|c|c|c|c|c|c|c|}
\hline \multirow{3}{*}{$\begin{array}{l}\text { Wawe- } \\
\text { lengths }\end{array}$} & \multirow{2}{*}{\multicolumn{2}{|c|}{ LF-Lpper Bed }} & \multicolumn{4}{|c|}{ First ILP } & \multirow{2}{*}{\multicolumn{2}{|c|}{$\begin{array}{c}\text { Second IID } \\
\text { Algo-B }\end{array}$}} \\
\hline & & & \multicolumn{2}{|c|}{ Algo-B } & \multicolumn{2}{|c|}{$\mathrm{Al}_{20}-\mathrm{A}$} & & \\
\hline & NSF & EON & NSF & FON & NSF & EON & NSF & EON \\
\hline 10 & 198 & 255 & 150 & 250 & in & $2 n 2$ & Iडा & 227 \\
\hline II & 178 & 301 & 170 & 265 & 184 & 274 & 165 & 249 \\
\hline 12 & 218 & 317 & 187 & 278 & 195 & 284 & 174 & 272 \\
\hline 13 & 205 & 329 & 195 & 290 & 205 & 295 & 183 & 269 \\
\hline 14 & 283 & 337 & 214 & 906 & 215 & 310 & 155 & 300 \\
\hline 15 & 243 & 344 & 2226 & 314 & 223 & 316 & 2003 & 305 \\
\hline 16 & 253 & 350 & 239 & 318 & 233 & 319 & 216 & 314 \\
\hline 17 & 263 & 396 & 250 & 325 & 253 & 335 & 229 & 327 \\
\hline 18 & 267 & 362 & 255 & 334 & 242 & 339 & 229 & 329 \\
\hline 19 & 265 & 367 & 263 & 337 & 254 & 340 & 250 & 336 \\
\hline 20 & 268 & 370 & 265 & 340 & 256 & 341 & 250 & 343 \\
\hline 21 & 268 & 373 & 267 & 347 & 261 & 37 & $25 ?$ & 345 \\
\hline 22 & 268 & 374 & 267 & 352 & 264 & 355 & 290 & 347 \\
\hline 23) & 208 & 374 & 268 & 361 & 268 & 361 & 261 & 361 \\
\hline 24 & 268 & 374 & - & 364 & - & 364 & 264 & 357 \\
\hline 25 & 268 & 374 & - & 367 & . & 370 & 265 & 367 \\
\hline
\end{tabular}

fractional solutions may have no criterion for choosing one path over another. Hence we may end up picking nonoptimal paths and wavelengths to satisfy the demand of a source-destination pair. If undesired paths and wavelength are picked then the heuristics were observed not to converge to the total number of connections even if we had large number of wavelengths available. Hence the first conclusion is that we do not to solve the relaxed problem with number of wavelengths greater than the LP lower bound (19 and 22 for NSFNET and EONNET networks). More details of various algorithms are available in [4].

We notice that algorithms-B which inputs solutions obtained by using the second ILP has higher blocking in all cases. This is due to the fact that the second ILP has fewer constraints and so the rounding heuristic has many equally promising choices for choosing routes and wavelengths to satisfy the demand. When the network has large number of connections and large number of nodes it was our computational experience that solving LP-relaxation of the second ILP occupies less memory and less computational time than solving LP-relaxation of the first ILP. The second conclusion we draw is to solve LP-relaxation of the first ILP and then use algorithm-A for moderately large networks, and for large networks solve LP relaxation for the second ILP and use algorithm-C.

\section{REFERENCES}

[1] R. Ramaswami and K. N. Sivarajan, "Routing and wavelength assignment in all-optical networks," IEEE/ACM Trans. n Networking, pp. 489-500, Oct. 1995.

[2] R. M. Krishnaswamy and K. N. Sivarajan, "Design of logical topologies: A linear formulation for wavelength routed optical network with no wavelength changers," IEEE/ACM Trans. Networking, pp. 186-198, Apr. 2001.

[3] D. Banerjee and B. Mukherjee, "Practical approaches for routing and wavelength assignment in large all-optical wavelength routed networks," IEEE J. Select. Areas Commun., vol. 14, 1996.

[4] R. M. Krishnaswamy, "Algorithms for routing wavelength assignment and topology design in optical networks," Ph.D. dissertation, Dept. of Electrical Commun. Eng., Indian Institute of Science, , Bangalore, India, Nov. 1998.

[5] M. J. O'Mahony, D. Simeonidu, A. Yu, and J. Zhou, "The design of the European optical network," J. Lightwave Technol., vol. 3, pp. 817-828, May 1995.

[6] S. Baroni, P. Bayvel, and R. J. Gibbens, "On the number of wavelengths in arbitrarily-connected wavelength-routed optical networks," Optical Society of America, TOPS, vol. 20, pp. 195-204, 1998. 